\title{
Extracellular Proteins Limit the Dispersal of Biogenic Nanoparticles
}

4

6

$9{ }^{2}$ Glenn T. Seaborg Institute, Lawrence Livermore National Laboratory, Livermore, CA, 10

$11{ }^{3}$ Advanced Light Source, Lawrence Berkeley National Laboratory, Berkeley, CA, USA

$12{ }^{4}$ Earth Science Division, Lawrence Berkeley National Laboratory, Berkeley, CA, USA

$13 \quad{ }^{5}$ Department of Environmental Science, Policy, and Management, University of

$17 *$ Current Address: United States Geological Survey Water Resources Division, 8505

18 Research Way, Middleton, WI, USA 53562

$19+{ }^{+}$Corresponding author: jwmoreau@usgs.gov 
1 Summary: Proteins are trapped within dense aggregates of bacterially formed metal-

2 sulfide nanoparticles, and may have played a key role in the aggregation process.

4 High spatial-resolution secondary ion microprobe spectrometry, synchrotron

5 radiation Fourier-transform infrared spectroscopy and polyacrylamide gel analysis

6 demonstrate the intimate association of proteins with spheroidal aggregates of

7 biogenic zinc sulfide nanocrystals, an example of extracellular biomineralization.

8 Experiments involving synthetic $\mathrm{ZnS}$ nanoparticles and representative amino acids

9 indicate a driving role for cysteine in rapid nanoparticle aggregation. These

10 findings suggest that microbially-derived extracellular proteins can limit dispersal

11 of nanoparticulate metal-bearing phases, such as the mineral products of

12 bioremediation, that may otherwise be transported away from their source by

13 subsurface fluid flow.

\section{Introduction}

16 Sulfate-reducing bacteria can lower the concentrations of metals in anoxic waters by

17 sequestering metals into nanoparticles (e.g., 1-3). However, these particles are

18 potentially highly mobile, due to their small size (4), and can redissolve quickly if

19 conditions change (5). Sulfide nanoparticles may be less than $2 \mathrm{~nm}$ in diameter,

20 comparable in size to aqueous molecular clusters (6); most are 2 to $6 \mathrm{~nm}(2,7)$.

21 Aggregation can restrict nanoparticle transport by inducing settling (8,9), and drive

22 crystal growth (leading to decreased solubility) (cf. 10, 11). Some organics can promote

23 aggregation. Amine-bearing molecules, for example, have been shown to organize 
1 sulfide nanoparticles into semiconductor nanowires (12). In this study, we investigated

2 the hypothesis that natural organic matter contributes to the formation of densely

3 aggregated nanoparticulate $\mathrm{ZnS}$ spheroids, and is preserved in nanometer-scale pores (7).

4 We use microanalytical and direct isolation approaches to analyze nanoparticle

5 aggregates formed in natural sulfate-reducing bacterial biofilms $(7,13)$. We also

6 experimentally evaluate the potential for various amino acids to induce rapid aggregation

7 of metal-sulfide nanoparticles.

8 We examined sulfate-reducing bacteria-dominated biofilms collected from the

9 Piquette lead and zinc mine, a flooded system $\left(\mathrm{pH} \sim 7, \mathrm{~T}^{\circ} \mathrm{C} \sim 8\right)$ in southwestern

10 Wisconsin, USA (13). Ultramicrotomed biofilm sections that contain spheroidal

11 aggregates of biogenic ZnS nanoparticles (Figs. S1-S4) were imaged with transmission

12 electron microscopy (TEM), prior to in situ elemental microanalysis with secondary ion

13 mass spectrometry at a spatial resolution of $\sim 50 \mathrm{~nm}$ (NanoSIMS) (14). Nitrogen in the

14 samples is detected by NanoSIMS as $\mathrm{CN}^{-}, \mathrm{NO}^{-}$, and $\mathrm{NS}^{-}$secondary ions, and is quantified

15 by comparison to reference samples $(14,15)$.

16 Comparison of TEM images with NanoSIMS sulfur distribution maps

17 demonstrates that $\mathrm{ZnS}$ spheroids are the only structures within the biofilm that contain

18 significant $\mathrm{S}$ concentrations (Figs. 1A, C). The composite NanoSIMS data show the

19 intimate association of $\mathrm{N}$ with biofilm ZnS (Figs. 1A, C); $\mathrm{N}$ is present throughout these

20 aggregates at significantly higher levels than in abiotic $\mathrm{ZnS}$ reference materials (Figs. 1D,

21 E). Pores in the $\mathrm{ZnS}$ spheroids appear as low diffraction-contrast features in TEM

22 images, due to a lower concentration of sphalerite nanoparticles (Fig. S2). Porous

23 regions are associated with the highest $\mathrm{N}$ concentrations (Figs. 1B, C). Nitrogen 
1 concentration measurements for individual spheroids varied by $14 \%$ (relative standard

2 deviation, $\mathrm{n}=134$ ), compared to an average measurement precision of $4 \%$, for individual

$3 \mathrm{ZnS}$ aggregates with an average diameter of $700 \mathrm{~nm}$. We estimate an average nitrogen

4 concentration for all analyzed $\mathrm{ZnS}$ spheroids of $1.6 \mathrm{wt} \%$, with a $95 \%$ confidence interval

5 of 0.8 to $3 \mathrm{wt} \%$ (14). By comparison, the average nitrogen concentration of synthetic

$6 \mathrm{ZnS}$ aggregates is approximately 100 times lower.

$7 \quad$ The small nitrate concentration of mine water $(\sim 3 \mu \mathrm{g} / \mathrm{g})$ is removed from the

8 biofilm during sample processing, and was therefore not expected to be the source of $\mathrm{N}$ in

$9 \mathrm{ZnS}$. To test this prediction, we analyzed the spheroids for $\mathrm{NO}^{-}$relative to $\mathrm{CN}^{-}(14)$. The

$10 \mathrm{CN}^{-} / \mathrm{NO}^{-}$ratio for a reference sample of $\mathrm{KNO}_{3}$ dissolved in graphite (14) ranges from <1

11 to 200 , with a median ratio of approximately 6 . The average $\mathrm{CN}^{-} / \mathrm{NO}^{-}$ratio of bacterial

12 spores, an organic nitrogen reference, was $2950 \pm 520$ (s.d.). The average $\mathrm{CN}^{-} / \mathrm{NO}^{-}$ratio

13 of the biofilm $\mathrm{ZnS}$ was $3300 \pm 870$ (s.d.). Measurement precision for $\mathrm{CN}^{-} / \mathrm{NO}^{-}$in the

14 biofilm $\mathrm{ZnS}$ is similar to sample variability because of low $\mathrm{NO}^{-}$secondary ion intensities.

15 Based on these analyses, we conclude that nitrogen in the biofilm $\mathrm{ZnS}$ is present neither

16 as nitrate nor nitrite, and is therefore organic in nature (14). This conclusion is further

17 supported by the presence of amide absorption features in the infrared spectra discussed

18 below. We note that from the average $\mathrm{N}$ content of $\mathrm{ZnS}$ estimated above, and taking an

19 average amino acid $\mathrm{N}$ concentration of approximately $11 \mathrm{wt} \%$, the $\mathrm{ZnS}$ spheroids contain

20 approximately $14 \mathrm{wt} \%$ amino acids.

21 Areas with cell-like morphologies enriched in N (Fig. 1) and P (Fig. S8) are

22 interpreted as being either whole or degraded microbial cells. These features are

23 morphologically distinct compared to $\mathrm{ZnS}$ spheroids, arguing against spheroid formation 
1 by nanoparticle encrustation and infilling of cells. We infer that the spheroids formed by

2 aggregation of biogenic $\mathrm{ZnS}$ nanoparticles (13) with extracellular polypeptides or

3 proteins. This process may have involved adsorption of amino-acids or peptides on to

4 nanoparticle surfaces (e.g., 16), or co-aggregation of protein molecules and nanoparticles.

5 High $\mathrm{N}$ concentrations along the surfaces of some aggregates suggest protein-rich regions

6 (Figs. 1B, C).

7 Synchrotron-based Fourier-transform infrared spectroscopy (SR-FTIR), with

8 roughly $10 \mu \mathrm{m}$ spatial resolution, was used to characterize organics associated with

9 biofilm $\mathrm{ZnS}$ aggregates (14). SR-FTIR analysis revealed that absorptions at

10 approximately $1580 \mathrm{~cm}^{-1}$ and $1640 \mathrm{~cm}^{-1}$ are only associated with ZnS spheroid-rich

11 regions of the biofilm (Fig. 2). These absorption features are well described for the amide

12 II and amide I vibration modes, respectively, and characteristic of polypeptide- and/or

13 protein-derived amino acids (17). Analyses of the spheroid-rich regions of biofilm varied

14 by a few percent in the relative magnitudes of amide I and II absorptions. The SR-FTIR

15 data confirm that the $\mathrm{N}$ detected by NanoSIMS analysis of spheroids is organic, and

16 support an origin in polypeptides or proteins.

17 Proteins were directly extracted from density-separated fractions dominated by

18 either organic biofilm components or $\mathrm{ZnS}$ spheroids (14; Fig. S6). Proteins from the

19 biofilm fraction produce faint bands when reacted with protein-specific stains in $4-10 \%$

20 polyacrylamide gels at molecular weights of $\sim 37 \mathrm{kDa}$ and $\sim 48 \mathrm{kDa}$ (14; Fig. 3). In

21 contrast, the $\mathrm{ZnS}$-enriched fraction yielded a strong band at $\sim 37 \mathrm{kDa}$, suggesting that the

22 nitrogen detected by NanoSIMS and SR-FTIR is associated with protein(s) of this

23 molecular mass. It was not possible to characterize the protein(s) further due to their low 
1 concentration and biofilm sample accessibility (13). However, we note that the observed

2 mass lies within the mass range of bacterial proteins known to bind certain metals (e.g.,

3 18-20), and genes for these proteins have been reported in some sulfate-reducing bacteria

4 (cf. 21-23). We speculate, therefore, that the $\mathrm{ZnS}$-associated protein(s) found in this

5 study may serve a metal-binding function.

6 In some aggregates, NanoSIMS data indicate overlapping $\mathrm{N}$ and $\mathrm{S}$ distributions,

7 implying the presence of fine-scale mixtures of $\mathrm{ZnS}$ nanoparticles and protein-rich

8 organic matter. Known bacterial metalbinding proteins bind zinc and other potentially

9 toxic metals (e.g., $\mathrm{Cd}, \mathrm{Cu}$ ), primarily at cysteine residues in proximity to $\mathrm{OH}^{-}$groups

10 (24). Experimental evidence shows that cysteine also binds strongly to ZnS nanoparticles

11 and limits their size to $<\sim 5 \mathrm{~nm}(25)$, and thiol groups bind strongly with sulfur-deficient

12 surface $\mathrm{Fe}(\mathrm{II})$ atoms in pyrite $\left(\mathrm{FeS}_{2}\right)(16)$. The conditional stability constant for mono-

13 ligand cysteine- $\mathrm{Zn}^{2+}$ complexation in low ionic strength solutions $\left(=0.1 \mathrm{M}_{c}\right)$ at $20-25^{\circ} \mathrm{C}$

14 is more than 4 orders of magnitude larger than those of all the other amino acids tested

15 except for lysine, for which the constant is about 2 orders of magnitude larger (14, Table

$16 \mathrm{~S} 1)$. These observations suggest that cysteine or cysteine-rich polypeptides or proteins

17 could have played a role in determining the $\mathrm{ZnS}$ particle size and aggregation state.

18 We tested the efficacy of individual amino acids $(100 \mu \mathrm{M})$ to promote

19 aggregation of synthetic $3 \mathrm{~nm}$ or smaller $\mathrm{ZnS}(10 \mu \mathrm{M})(14)$. The chosen amino acids

20 (alanine, aspartate, cysteine, lysine, phenylalanine, proline, and serine) possess

21 chemically distinct side-chain functional groups. Aggregation was monitored

22 periodically using dynamic light scattering (DLS; 14), and results showed that the

23 inorganic aggregation of $\mathrm{ZnS}$ at first occurred rapidly to form $\sim 100 \mathrm{~nm}$-diameter 
1 aggregates, but then slowed greatly or ceased after one week (Figs. 4, S7). In contrast,

$2 \mathrm{ZnS}$ nanoparticles in the presence of cysteine exhibited more extensive and prolonged

3 aggregation, ultimately forming 1-10 $\mu \mathrm{m}$-sized structures. Other amino acids had little

4 (e.g., serine) to no (e.g., proline) detectable effect on nanoparticle aggregation relative to

5 controls (Fig. S7). Cysteine in the absence of $\mathrm{ZnS}$ formed no measurable aggregates

6 (Fig. 4), and humic compounds added to $\mathrm{ZnS}$ nanoparticle suspensions did not accelerate 7 aggregation.

8 The DLS results correlate with prior studies of the adsorption of amino acids,

9 other organic ligands, and inorganic ions to the surfaces of metal chalcogenides $(16,26$,

10 27). For example, the sulfhydryl group present in cysteine and mercapto- compounds

11 exhibits strong specific binding to the surfaces of sulfide minerals and nanoparticles.

12 Similarly, serine with a terminal hydroxyl group causes somewhat more aggregation than

13 observed in control samples, as was expected from both the weaker chemical interaction

14 of this group with sulfide surfaces and the higher $\mathrm{pK}_{\mathrm{a}}$ relative to cysteine (9.15 vs. 8.33).

15 Thus, we conclude that strong specific chemical binding is a necessary prelude to amino

16 acid- or protein-driven $\mathrm{ZnS}$ nanoparticle aggregation.

17 Mineral-protein mixtures with internal organization are typically considered

18 biominerals, and biominerals normally form within orga nisms. The structures reported

19 here represent an exception to this pattern. Proteins, peptides, and amino acids could be

20 released after cell death and scavenged by hydrophobic $\mathrm{ZnS}$ surfaces. Alternatively,

21 bacteria may export Zn-binding proteins for a physiological reason. Most known

22 bacterial metal-binding proteins are produced for intracellular binding and subsequent

23 export of toxic metals (24). In the case reported here, biofilm proteins may bind excess 
1 aqueous $\mathrm{Zn}^{2+}$, or interact with bound $\mathrm{Zn}(\mathrm{II})$ and other proteins subsequent to $\mathrm{ZnS}$

2 precipitation. In either scenario, the aggregation of metalsulfide nanoparticles was

3 promoted, preventing incidental uptake by (e.g., 28, 29) or entombment of cells. From

4 the dense, spheroidal morphologies, the rate of aggregation appears to be reaction-limited

5 (30). Similar densely packed aggregates of biogenic metal-bearing nanoparticles have

6 been reported from other metal-contaminated systems (e.g., 31). Such aggregates in

7 sediments could trap and possibly preserve organic molecules or their degradation

8 products in sediments or rocks.

9 Microbial and chemical redox transformations of metals can result in the 10 precipitation of metal-bearing nanoparticles across a range of environmental conditions

11 (32). The aggregation state of these particles may have a strong impact on metal mobility

12 and water quality (33). Our results suggest that aggregation induced by extracellular

13 metal-binding polypeptides and proteins plays an important role in limiting nanoparticle

14 dispersal in natural environments.

15

16

17

\section{References}

1. J.R. Lloyd, A.N. Mabbett, D.R. Williams, L.E. Macaskie, Hydromet. 59, 327-337 (2001).

2. Y. Suzuki, S.D. Kelly, K.M. Kemner, J.F. Banfield, Nature 419, 134 (2002).

3. S.L. Hockin, G.M. Gadd, Appl. Environ. Microbiol. 69, 7063-7072 (2003).

4. B.D. Honeyman, Nature 397, 23-24 (1999).

5. J.G. Hering, W. Stumm, In M.F. Hochella, Jr., A.F. White (Eds.), Rev. Min. Geochem. 23, 427-465 (1990).

6. G.W. Luther, S.M. Theberge, D.T. Rickard, Geochim. Cosmochim. Acta 63, 31593169 (1999). 
7. J.W. Moreau, R.I. Webb, J.F. Banfield, Amer. Min. 89, 950-960 (2004).

8. C. Allain, M. Cloitre, F. Parisse, J. Colloid Interf. Sci. 178, 411-416 (1996).

9. S.A. Bradford, S.R. Yates, M. Bettehar, J. Simunek, Wat. Res. Res. 38, 1327, doi: 10.1029/2002WR001340 (1996).

10. J.W. Zhang, G.H. Nancollas, In M.F. Hochella, Jr., A.F. White (Eds.), Rev. Min. Geochem. 23, 365-393 (1990).

11. W. Stumm, J.J. Morgan, Aquatic chemistry: chemical equilibria and rates in natural waters (Wiley and Sons, New York, 1996), pp. 400-414.

12. Q. Lu, F. Gao, D. Zhao, Nanotech. 13, 741-745 (2002).

13. M. Labrenz et al., Science 290, 1744-1747 (2000).

14. Materials and methods are available as supporting material on Science Online.

15. E.H. Hauri, J. Wang, D.G. Pearson, G.P. Bulanova Chem. Geol. 185, 149-163 (2002).

16. J. Bebie, M.A.A. Schoonen, Geochem. Trans 8 (2000).

17. H.H. Mantsch, D. Chapman, Infrared spectrosc. biomol., (Wiley-Liss, New York, 1996).

18. M.B. Khazaeli, R.S. Mitra, Appl. Environ. Microbiol. 41, 46-50 (1981).

19. E. Kurek, A.J. Francis, J.M. Bollag, Arch. Environ. Contam. Toxicol. 20, 106-111 (1991).

20. D.H. Nies, J. Bact. 174, 8102-8110 (1992).

21. N. Naz, H.K. Young, N. Ahmed, G.M. Gadd, Appl. Environ. Microbiol. 71, 46104618 (2005).

22. T.A.M. Bridge, C. White, G.M. Gadd, Microbiol. 145, 2987-2995 (1999).

23. C.A. Blindauer et al., PNAS 98, 9593-9598 (2001).

24. B.P. Rosen, J. Biol. Inorg. Chem. 1, 273-277 (1996).

25. C.L. Torres-Martinez et al., Nanotech. 10, 340-354 (1999). 
26. L. Ronngren, S. Sjoberg, Z. Sun, W. Forsling, P.W. Schindler, J. Colloid Interf. Sci. 145, 396-404 (1991).

27. W. Vogel, Langmuir 16, 2032-2037 (2000).

28. T. Barkay, J. Schaefer, Curr. Opin. Microbiol. 4, 318-323 (2001).

29. J.A. Kloepfer, R.E. Mielke, J.L. Nadeau, Appl. Environ. Microbiol. 71, 2548-2557 (2005).

30. G.A. Waychunas, In J.F. Banfield, A. Navrotsky (Eds.) Rev. Min. Geochem. 44, 105166 (2001).

31. J.S. Ahn, Y.S. Park, J.Y. Kim, K.W. Kim, Environ. Geochem. Health 27, 147-157 (2005).

32. M.F. Hochella, Jr., Geochim. Cosmochim. Acta 66, 735-743 (2002)

33. M.S. Diallo, N. Savage, J. Nanopart. Res. 7, 325-330 (2005).

34. We thank Tamara Thomsen and colleagues of Diversion SCUBA (Madison, WI) for recovering biofilm samples; Rick and Robyn Webb (University of Queensland, Australia), Hengzhong Zhang (University of California, Berkeley), and Christina Ramon (Lawrence Livermore National Laboratory, LLNL) for assistance in preparing biofilm and synthetic ZnS samples for TEM and NanoSIMS; Larry Nittler (Carnegie Institution of Washington, D.C.) for assistance with processing NanoSIMS data; Robert G. Wilson for data on nitrogen in $\mathrm{ZnS}$; and Michael Thelen and Chris Jeans (LLNL) for assistance with protein extractions. The comments of two anonymous reviewers greatly improved the quality and clarity of our manuscript. Work was funded by the U.S. Department of Energy Basic Energy Sciences Program under Contract no. DE-FG02-04ER15507, the NASA Astrobiology Institute under Contract no. NNA04CC02A (JWM and JFB), and the U.S. Department of Energy Office of Biological and Environmental Research Genomics: GTL research program (PKW and IDH). Work was performed at LLNL under the auspices of the U.S. Department of Energy under Contract no. W-7405-Eng-4. The Advanced Light Source is supported by the Director, Office of Science, Office of Basic Energy Sciences, of the U.S. Department of Energy under Contract no. DE-AC02-05CH11231. 


\section{Figure Captions:}

Figure 1: NanoSIMS secondary ion images showing $\mathrm{C}, \mathrm{N}$ and $\mathrm{S}$ distributions in an ultramicrotomed TEM section of biofilm. (A) Composite element distribution map $(\sim 10 \mu \mathrm{m} \times 10 \mu \mathrm{m})$ of ${ }^{12} \mathrm{C}$ (blue), ${ }^{12} \mathrm{C}^{14} \mathrm{~N}$ for $N$ (green), and ${ }^{32} \mathrm{~S}$ (red). Colors reflect the proportion each species. For example, uniformly red regions represent relatively pure ${ }^{32} \mathrm{~S}$ (as $\mathrm{ZnS}$ ), while orange or yellow regions indicate the presence of increased levels of nitrogen. Cyan regions indicate the presence of both carbon and nitrogen, with little to no sulfur (no $\mathrm{ZnS}$ ). (B) TEM image of several conjoined $\mathrm{ZnS}$ spheroidal aggregates. (C) NanoSIMS composite element distribution map of (B). (D) NanoSIMS composite element distribution map of ultramicrotomed Balmat ZnS. (E) NanoSIMS composite element distribution map of synthetic nanoparticulate $\mathrm{ZnS}$. (F) Color box plots of the relative ion abundances displayed in (A, C, D and E). Primary colors and maximum ion counts are noted for each species along each axis; all axes are linear with respect to ion counts. In the left box ("binary"), only binary ion compositions (one or two species) are shown; in the right box ("ternary"), only primary (the species corresponding to each axis) and ternary ion compositions (three species) are shown. Black and white "corners" correspond to points of minimum and maximum ion counts, respectively, for all three species. All scale bars are $1 \mu \mathrm{m}$. Figs. S8-S10 (14) present grayscale versions of Figs. $1 \mathrm{~A}, \mathrm{C}$, and D, respectively.

Figure 2. SR-FTIR transmission spectra of biogenic $\mathrm{ZnS}$ aggregates (black) and background biofilm (gray). Amide I $\left(\sim 1640 \mathrm{~cm}^{-1}\right)$ and II $\left(\sim 1580 \mathrm{~cm}^{-1}\right)$ absorption features are diagnostic of amino-acid associated bond vibrations in polypeptides and/or proteins.

Figure 3: Composite SDS-PAGE gel electrophoresis image of biofilm and $\mathrm{ZnS}$ protein extractions. SyproOrange and colloidal silver molecular weight standards (far left and right lanes, respectively). (A) Extraction from the biofilm organic fraction stained with SyproOrange. (B) Extraction of the $\mathrm{ZnS}$ spheroid fraction stained with SyproOrange. (C and D) Replicate extractions of biofilm organic fraction stained with silver. (E and F) Replicate extractions of $\mathrm{ZnS}$ spheroids fraction stained with silver. Numbers are molecular masses in kilodaltons $(\mathrm{kDa})$.

Figure 4: Size distribution curves from dynamic light scattering (DLS) data acquired in $\mathrm{ZnS}$ nanoparticles aggregation experiments. (A) Control experiments. 10 $\mathrm{uM} \mathrm{ZnS}$ nanoparticles alone (solid lines) aggregate within 1 day to form $\sim 100 \mathrm{~nm}$ radius clusters that exhibit little further growth over the 5 day period. $100 \mu \mathrm{M}$ cysteine alone (dashed lines) gives a very weak DLS signal with is no consistent trend in size distribution. (B) In the presence of both $10 \mu \mathrm{M} \mathrm{ZnS}$ and $100 \mu \mathrm{M}$ cysteine, sustained aggregation occurs over the 7 day period, resulting in aggregates that are more than 1 order of magnitude larger than the initial clusters. DLS correlation functions from which size distributions were derived are shown in Fig. S7. 
Supplementary Materials and Methods:

\section{Transmission electron microscopy (TEM)/Secondary ion mass spectrometry (SIMS) biofilm sample preparation:}

Samples of biofilm were fixed with $4 \%$ glutaraldehyde, as described previously (S1). TEM images (Fig. S4) confirm that, because the sectioning resin neither displaces nor damages fine-scale biofilm features, ultramicrotomed samples of biofilm used for TEM imaging were also suitable for SIMS analyses.

\section{Secondary Ion Mass Spectrometry (SIMS):}

SIMS was performed using the Lawrence Livermore National Laboratory NanoSIMS 50 (Cameca, Gennevilliers Cedex, France). The measurements were made with a 0.2 to 0.7 pA, $16 \mathrm{keV}^{133} \mathrm{Cs}^{+}$primary ion beam focused into a 50 to $100 \mathrm{~nm}$ diameter spot, rastered over sample areas of $\sim 10 \times 10 \mu \mathrm{m}^{2}$. The isotope imaging measurements consist of 15 to 100 replicate scans of 256 x 256 or 512 x 512 pixels with dwell times of 1-2 ms/pixel Secondary ion intensities were collected simultaneously in multi-collection mode using three different collector configurations, $\left[{ }^{12} \mathrm{C}^{-},{ }^{12} \mathrm{C}^{14} \mathrm{~N}^{-},{ }^{31} \mathrm{P}^{-},{ }^{32} \mathrm{~S}^{-}\right],\left[{ }^{12} \mathrm{C}^{-},{ }^{12} \mathrm{C}^{14} \mathrm{~N}^{-},{ }^{14} \mathrm{~N}^{16} \mathrm{O}^{-}\right.$, $\left.{ }^{32} \mathrm{~S}^{-},{ }^{14} \mathrm{~N}^{32} \mathrm{~S}^{-}\right]$and $\left[{ }^{12} \mathrm{C}^{-},{ }^{12} \mathrm{C}^{14} \mathrm{~N}^{-},{ }^{32} \mathrm{~S}^{-},{ }^{14} \mathrm{~N}^{32} \mathrm{~S}^{-}\right]$. A mass resolving power of $\sim 4000$ was used. Samples were also simultaneously imaged using secondary electrons. The ${ }^{12} \mathrm{C}^{14} \mathrm{~N}^{-}$ $/{ }^{14} \mathrm{~N}^{16} \mathrm{O}^{-}$ratio in the biofilm and $\mathrm{ZnS}$ spheroids was compared to this ratio in a sample of $\mathrm{KNO}_{3}$ dissolved in graphite as a nitrate reference $\left(\sim 0.3 \mathrm{wt} \% \mathrm{KNO}_{3}\right)$, and bacterial spores as an organic nitrogen reference, to test for significant levels of nitrate in the $\mathrm{ZnS}$ spheroids. Nitrogen concentration in the $\mathrm{ZnS}$ aggregates was estimated based on a relative sensitivity factor for $\mathrm{NS}^{-}\left(\mathrm{RSF}_{\mathrm{NS}}\right)$ of $4.2 \times 10^{24} \mathrm{~cm}^{-3}$ for $\mathrm{NS}^{-}$relative to $\mathrm{S}^{-}$in $\mathrm{ZnS}$ (S2). The ZnS standard was produced by implanting $\mathrm{N}$ into a $\mathrm{ZnS}$ crystal with $150-\mathrm{keV}$ at a fluence of $1 \times 10^{14}$ atoms $/ \mathrm{cm}^{2}$. The abundance of $\mathrm{N}$ (atoms per $\mathrm{cm}^{3}$ ) in $\mathrm{ZnS}\left(\mathrm{C}_{\mathrm{N}}\right)$ is estimated from:

$$
\mathrm{C}_{\mathrm{N}}=\mathrm{RSF}_{\mathrm{NS}} * \mathrm{I}_{\mathrm{NS}} / \mathrm{I}_{\mathrm{S}}
$$

where $\mathrm{I}_{\mathrm{NS}}$ and $\mathrm{I}_{\mathrm{S}}$ are ion count rates for $\mathrm{NS}^{-}$and $\mathrm{S}^{-}$corrected for the abundances of ${ }^{14} \mathrm{~N}$ and ${ }^{32} \mathrm{~S}$ relative to total $\mathrm{N}$ and $\mathrm{S}(\mathrm{S} 3)$. The abundance of $\mathrm{N}$ in $\mathrm{ZnS}$ (wt\%) is calculated from $\mathrm{C}_{\mathrm{N}}$, Avogadro's number, the atomic mass of $\mathrm{N}$, and the density of $\mathrm{ZnS}$. The estimate of absolute abundance of $\mathrm{N}$ in the $\mathrm{ZnS}$ aggregates is given a factor of two uncertainty to allow for the $\mathrm{RSF}_{\mathrm{NS}}$ being generated on a different SIMS instrument; actual accuracy is likely higher. Data for $\mathrm{CN}^{-}$are used in the figures because $\mathrm{CN}^{-}$has approximately 100 times higher intensity than $\mathrm{NS}^{-}$(Fig. S11) and therefore provides higher clarity images.

40

41 Composite multi-element images were constructed using Photoshop (Adobe) and the 42 NanoSIMS data analysis software, L'image (developed by L. Nittler, Carnegie Institution 43 of Washington, Washington, D.C., USA). Grey-scale ${ }^{12} \mathrm{C}^{-},{ }^{12} \mathrm{C}^{14} \mathrm{~N}^{-}$and ${ }^{32} \mathrm{~S}^{-}$intensity 44 images generated by L'image (e.g. Figs. S8-S10) were assigned to blue, green and red 45 channels, respectively, in Photoshop RGB (red/green/blue) image files. Therefore, 
1 primary colors in the images represent the presence of only one of the elements, whereas 2 "binary" or "ternary" colors represent mixtures of two or three elemental components, 3 respectively. Black indicates areas of no data (i.e. holes in the TEM section). The color 4 box plot "key" to Fig. 1 (F) represents in three-dimensional space the surfaces of possible 5 binary and ternary mixtures of the three colors (i.e. elements). The significance of 6 measurements obtained via NanoSIMS analyses of biofilm ZnS, for $>1,000$ regions-ofinterest (ROIs) of approximately $180 \mathrm{~nm}^{2}$ each, was determined by comparison to NanoSIMS analyses of natural and synthetic $\mathrm{ZnS}$ reference materials (Figs. 1D, E). In addition to a natural single-crystal ZnS standard (Balmat sphalerite, NBS123) (Fig. 1D), nanoparticulate ZnS synthesized (S4) by H. Zhang (UC Berkeley) (Fig. 1E) was used to represent a similarly structured (i.e., nanocrystalline) pure $\mathrm{ZnS}$ reference material for comparison.

In the course of analysis by dynamic SIMS methods such as NanoSIMS, the high energy primary ion beam breaks chemical bonds in the sample. Dimers produced in the course of primary beam sputtering originate from atoms in the sample, and therefore, at a minimum, it is possible to determine if elements are associated with each other at the scale of the primary beam. To some extent, dimers will come from bonded atoms or be affected by chemical bonding in the sample (S5). Therefore, we analyze organic and nitrate reference materials for $\mathrm{CN}^{-}$and $\mathrm{NO}^{-}$to determine empirically if the relative yield of these two dimers can be used to constrain the nature of $\mathrm{N}$ in the $\mathrm{ZnS}$ aggregates. The ${ }^{12} \mathrm{C}^{14} \mathrm{~N}^{-14} \mathrm{~N}^{16} \mathrm{O}^{-}$data for the organic standard and the biofilm $\mathrm{ZnS}$ are well constrained around 3000, within the range of counting statistics. The ${ }^{12} \mathrm{C}^{14} \mathrm{~N}^{-} /{ }^{14} \mathrm{~N}^{16} \mathrm{O}^{-}$data for the inorganic reference material, $\mathrm{KNO}_{3}$ dissolved in graphite, is more scattered and highly skewed (skewness $=3.2$ ), with over $75 \%$ of the data below a ratio of 20 and less than $10 \%$ over 50 (Fig. S5A). These data are from two $10 \times 10 \mu \mathrm{m}^{2}$ fields, subdivided into 169 ROIs each, and provide a reference for $\mathrm{NO}_{3}{ }^{-}$associated with carbon. The $\mathrm{NO}_{3}{ }^{-}$ abundance in the graphite is heterogeneous (mean of $\sim 0.3 \mathrm{wt} . \%$ ), providing a range of concentrations for comparison to other samples. The experiment shows that even in the case of intimate association of $\mathrm{NO}_{3}{ }^{-}$with carbon at low concentrations at sputtering equilibrium, the relative yield of $\mathrm{CN}^{-}$to $\mathrm{NO}^{-}$is at least a factor of 10 lower than observed in the organic sample and the biofilm $\mathrm{ZnS}$ aggregates (Fig. S5B). At higher concentrations relative to $\mathrm{C}$, the $\mathrm{CN} / \mathrm{NO}$ ratio is below 1 . We take this experiment as evidence that the $\mathrm{N}$ in the $\mathrm{ZnS}$ aggregates is not present as nitrate or nitrite, and therefore is organic in nature.

\section{Synchrotron-radiation Fourier transform infra-red spectroscopy (SR-FTIR):}

SR-FTIR is capable of detecting and differentiating amongst nucleic acids, lipids, amino acids, and polysaccharides (S6). The spatial resolution of SR-FTIR, coupled with optical microscopy, is diffraction limited, or between 2 and $10 \mu \mathrm{m}$ in the mid-infrared (S7). SRFTIR analyses were conducted on uncoated ultramicrotomed sections of the same biofilm samples used for NanoSIMS analyses. The location of the synchrotron probe relative to targets selected with optical microscopy was calibrated using IR-sensitive targets on 
standards. Background spectra were obtained and used as reference spectra for both samples and standards to remove atmospheric $\mathrm{H}_{2} \mathrm{O}$ and $\mathrm{CO}_{2}$ absorptions.

\section{Biofilm/ZnS separation and total protein extraction:}

Biofilm samples were homogenized by repeated pipetting action through a series of sterile syringe needles of increasingly smaller inner-bore diameter (18-, 21- and 22gauge, respectively, for approximately 20 minutes each). Homogenized biofilm slurries were then pelleted by centrifugation, re-suspended in lithium polytungstate solution (LST) of density $\sim 2.8 \mathrm{~g} \mathrm{~cm}^{-3}$ (Geoliquids, Prospect Heights, IL, USA), and then subjected to further low-speed centrifugation $(\sim 5 \mathrm{mins}$ at $1000 \mathrm{rpm})$ in autoclaved microcentrifuge tubes (Eppendorf, Hamburg, Germany). ZnS-rich biofilm fragments $\left(\rho \sim 4.1 \mathrm{~g} \mathrm{~cm}^{-3}\right)$ were easily pelleted at the bottom of LST-filled tubes during centrifugation, while ZnS-poor biofilm fragments rose to the top of the LST solution to be re-pipetted into new tubes. This process of low-speed centrifugation, density-based fractionation, collection and resuspension was repeated several times, until no further separation of $\mathrm{ZnS}$-rich and $\mathrm{ZnS}$ poor biofilm fractions was observed. Both fractions were transferred a final time by pipette to clean tubes of nuclease-free water, and then pelleted, washed and re-suspended 6-8 times, to remove all traces of LST. Each resulting fraction represents a subsample of biofilm or $\mathrm{ZnS}$ spheroids with greatly increased relative concentrations of organic matter or $\mathrm{ZnS}$, respectively. This method avoids the use of surfactants or solvents that might solubilize and destroy organic biomolecules, including nucleic acids and proteins. Each "density fraction" from the separation process was pelleted and re-suspended a final time in $2 \%$ sodium dodecyl sulfate (SDS) solution for one hour at room temperature with gentle mixing to denature any proteins $(S 8)$.

\section{Protein gel electrophoresis:}

Molecular weight standard "ladders" were run on both sides of all sample gel lanes to correct for horizontal distortions commonly produced during gel electrophoresis. Protein extractions were analyzed using two different molecular weight ladders and two different staining methods, for comparison of sample protein size/weights and staining efficiencies. One set of gels was reacted with a silver-based stain (BioRad, Hercules, CA, USA) (S9), and Kaleidoscope (BioRad) pre-stained molecular weight ladders were used to calibrate the weight/size of protein bands. These gels were imaged using a standard Epson scanner. The second set of gels was stained with SyproOrange (BioRad), a UV-fluorescent dye that specifically binds to proteins (S10), and protein bands were calibrated to broad-range SyproOrange molecular weight ladders. These gels were imaged using a UV-VIS light gel documentation system (BioRad). Both SyproOrange and silver staining can be used to detect $=10 \mathrm{ng}$ of protein.

\section{ZnS nanoparticle synthesis:}

Nano-ZnS was synthesized by the reaction of $150 \mathrm{mM} \mathrm{ZnCh}$ and aqueous sulfide, which were dissolved separately into $200 \mathrm{ml}$ of ultra-pure $(0.1 \mu \mathrm{m}$ pore-size double-filtered 
1 MilliQ; Millipore, Billerica, MA, USA) DI water and reacted drop wise under constant

2 stirring for $1.5 \mathrm{hr}(\mathrm{S} 5)$.

\section{ZnS nanoparticle/amino acid aggregation experiments:}

The initial size of $\mathrm{ZnS}$ precipitates was determined to be $=3 \mathrm{~nm}$ diameter using a UV-vis spectrometer (Ocean Optics, Dunedin, FL, USA) calibrated for ZnS particle size using published TEM observations (S11). The following experimental conditions were established in separate glass flasks: (a) "blanks" (ultrapure water only), (b) $\mathrm{ZnS}$ nanoparticles only, (c) a single amino acid only and (d) ZnS nanoparticles plus a single amino acid. All incubation flasks were (in the following order) detergent-washed, rinsed with MilliQ water three times, acid-washed in $0.1 \mathrm{~N} \mathrm{HCl}$ overnight, rinsed five times with MilliQ water, and sealed with parafilm, prior to the experiment. After the addition of any reagents, flasks were resealed with parafilm and kept sealed during the experiment between sampling times. All flasks were gently stirred throughout the experiment by acid-washed Teflon-coated magnetic stirrer bars. $1 \mathrm{ml}$ aliquots from each experimental condition were taken at $0,0.5,1,3,5$, and 7 days of reaction progress. Between sample measurements, $0.5 \mathrm{ml}$ of $0.1 \mathrm{~N} \mathrm{HCl}$ was flushed through the sample line and chamber, followed by $3 \mathrm{ml}$ of ultrapure water, to avoid cross-contamination of samples. Three measurements of $40-60$ accumulations each were obtained per analysis at each sampling time point.

The amino acids chosen for these aggregation experiments represent the following classes of structures and functional groups: alanine (nonpolar, aliphatic), aspartate (acidic, negatively charged), cysteine (polar, uncharged, thiol-bearing), lysine (basic, positively charged), phenylala nine (aromatic), proline (polar, uncharged, imine-bearing), and serine (polar, uncharged, hydroxyl-bearing). Their conditional stability constants for mono-ligand complexation of aqueous $\mathrm{Zn}^{2+}$ are given in Table $\mathrm{S} 1$, from data collected by Martell and Smith (S12).

\section{Dynamic light scattering (DLS) analysis:}

DLS analysis was performed with a PD-Expert Workstation (Precision Detectors, Bellingham, MA, USA) fitted with a quartz flow-through cell maintained at $25^{\circ} \mathrm{C}$. Laser light (685 $\mathrm{nm}$ wavelength) scattered through $135^{\circ}$ was sent via glass fiber to an optical correlator. The DLS correlation function is a direct measurement of the diffusion coefficient(s) of objects suspended in a solvent. For specific values of solvent viscosity and refractive index, an effective hydrodynamic radius, $R_{H}$, of the object(s) can be calculated (S13). In the present case, we observed light scattering from aggregates of $\mathrm{ZnS}$ nanoparticles. Because the exact shape and hydrodynamic properties of nanoparticulate aggregates are not well known, there may be systematic differences between the calculated $R_{H}$ and spatial dimensions observed by alternative techniques (such as electron microscopy). Determination of the distribution of $\mathrm{ZnS}$ aggregate sizes was performed using proprietary Precision Detectors software (PrecisionDeconvolve) that employs a regularization algorithm (S14) to seek a smooth, non-negative size distribution function that provides the best fit to the data. Low noise DLS data analyzed 
with this method can resolve multimodal dis tributions, provided the diffusion coefficients differ by greater than a factor of $\sim 2.5$.

Supplementary Figure Captions:

Figure S1: Field-emission scanning electron microscopy (FESEM) secondary electron image of micron-scale spheroidal aggregates of nanocrystalline biogenic $\mathrm{ZnS}$ formed in the biofilm

Figure S2: HRTEM image of cross-section through spheroidal biogenic $\mathrm{ZnS}$ aggregate in the biofilm. Multiple concentric rings (3-5 $\mathrm{nm}$ wide, shown by white arrows) of low electron density attributed to porous regions separate bands of densely aggregated nanoparticulate $\mathrm{ZnS}$.

Figure S3: HRTEM images of biogenic ZnS nanoparticles formed in a biofilm of sulfate-reducing bacteria. (A) The smallest $\mathrm{ZnS}$ nanoparticles are crystalline, as shown by $\{111\}$ lattice fringes in [02-2] projection, and <2 nm-diameter. (B) Several aggregated nanoparticles with boundaries indicated by dashed lines. (C) Disordered $\mathrm{ZnS}$ aggregates contain multiple stacking faults (interpolated along pairs of white lines) that give rise to wurtzite subdomains (across white line pairs) in sphalerite. (D) Example of aggregation producing faceted surfaces and negative crystals (dashed white triangle). All scale bars are $2 \mathrm{~nm}$.

Figure S4: TEM image of biofilm organic matter, cells and $\mathrm{ZnS}$ aggregates. Stained ultramicrotomed sections of biofilm show abundant $\mathrm{ZnS}$ spheroidal aggregates (black spheroidal features with strong diffraction contrast) surrounded by biofilm organic matter (white arrows), and in close proximity to cells (black arrows). Dark grey spheroidal features with lighter grey cores (grey arrows) are cells surrounded by capsular organic matter that has been stained with uranyl acetate and lead citrate. Scale bars are $1 \mu \mathrm{m}$.

Figure S5: ${ }^{12} \mathrm{C}^{14} \mathrm{~N}^{-14} \mathrm{~N}^{16} \mathrm{O}^{-}$ratios of $\mathrm{KNO}_{3}$ dissolved in graphite. (A) Histogram of the number versus ${ }^{12} \mathrm{C}^{14} \mathrm{~N}^{-14} \mathrm{~N}^{16} \mathrm{O}^{-}$ratio for each of $33810 \times 10 \mu \mathrm{m}^{2}$ regions analyzed. The distribution is highly skewed (skewness $=3.2$ ), with over $75 \%$ below a ratio of 20 and less than $10 \%$ over 50. (B) Plot of ${ }^{12} \mathrm{C}^{14} \mathrm{~N}^{-}{ }^{14} \mathrm{~N}^{16} \mathrm{O}^{-}$versus ${ }^{12} \mathrm{C}^{14} \mathrm{~N}^{-12} \mathrm{C}$ ratios for the same regions in (A). ${ }^{12} \mathrm{C}^{14} \mathrm{~N}^{-14} \mathrm{~N}^{16} \mathrm{O}^{-}$ratios are, for all analyses, at least one order of magnitude less than those observed in biofilm $\mathrm{ZnS}$ spheroids.

Figure S6: Microcentrifuge tubes of lithium-polytungstate solution (LST), biofilm and ZnS. The left-hand tube shows the color of pure LST. Homogenized biofilm is introduced and subjected to low speed centrifugation to separate lower and higher density fractions (middle tube). Density-driven separation is iteratively repeated until no further separation is achieved (right-hand tube). Material at the top of the LST solution consists predominantly of biofilm organic matter, while the pellet at the bottom is concentrated in ZnS spheroids. 
$1 \quad$ Figure S7: Dynamic light scattering (DLS) data acquired during ZnS nanoparticle 2 aggregation experiments. Top row: size distribution histograms. Bottom row: DLS 3 correlation functions. The first column shows the data for the control experiment in which ZnS nanoparticle aggregation was observed without any amino acid added. Subsequent columns are labeled with the name of the added amino acid. We investigated the ability of the amino acids to drive nanoparticle aggregation by comparing the timedependent cluster size with the no-amino acid control. The aggregation behavior in the presence of proline was indistinguishable from the control, and similar results were obtained for alanine and phenylalanine. Serine appeared to drive some additional aggregation relative to the control, and similar results were obtained for aspartate and lysine. Only experiments in the presence of cysteine showed extensive and prolonged aggregate formation.

Figure S8: NanoSIMS secondary ion images of biofilm with $\mathrm{ZnS}$ aggregates. Grayscale version of Fig. 1A in which each component element (i.e. color) is here shown separately as total counts. One additional frame has been added (lower right) to demonstrate the strong degree of phosphorus co-localization with only features exhibiting both increased carbon and nitrogen counts (upper row). Scale bars are $500 \mathrm{~nm}$.

Figure S9: NanoSIMS secondary ion images of biofilm with $\mathrm{ZnS}$ aggregates. Grayscale version of Fig. 1C in which each component element (i.e. color) is here shown separately as total counts. One additional frame has been added (lower right) to again demonstrate the absence of phosphorus in $\mathrm{ZnS}$ spheroids. Scale bars are $500 \mathrm{~nm}$.

Figure S10: NanoSIMS secondary ion images of ultramicrotomed Balmat ZnS reference standard. Grayscale version of Fig. 1D in which each component element (i.e. color) is here shown separately as total counts. Scale bars are $500 \mathrm{~nm}$.

Figure S11: NanoSIMS secondary ion images of biofilm with $\mathrm{ZnS}$ aggregates. Grayscale version of a different region of biofilm from the same sample shown in Fig. 1A. In addition to the same component elements shown in Fig. 1, the species ${ }^{32} \mathrm{~S}^{14} \mathrm{~N}$ is shown. Based on the significantly higher counts for ${ }^{12} \mathrm{C}^{14} \mathrm{~N}$ than for ${ }^{32} \mathrm{~S}^{14} \mathrm{~N}$, the former species was used to measure the nitrogen content of $\mathrm{ZnS}$ spheroids and other biofilm components.

Table S1: Stability constants for various amino acids. Data are taken from (12), for the reaction $\mathrm{M}+\mathrm{L} \rightarrow \mathrm{ML}$, where $\mathrm{M}=\mathrm{Zn}^{2+}$ and $\mathrm{L}=$ amino acid. $\mathrm{I}=$ ionic strength. 


\section{Supplementary References:}

1. J.W. Moreau, R.I. Webb, J.F. Banfield Amer. Min. 89, 950-960 (2004).

2. Written communication, Robert G. Wilson, December 30, 2006.

3. R.G. Wilson, F.A. Stevie, C.W. Magee, Secondary Ion Mass Spectrometry: A Practical Handbook for Depth Profiling and Bulk Impurity Analysis (Wiley, New York, 1989).

4. After the method of B.R. Müller, S. Majoni, R. Memming, D. Meissner, J. Phys. Chem. B 101, 2501-2507 (1997).

5. G. McMahon, H. Francois Saint-Cyr, C.J. Unkefer, C. Lechene, J. Am. Soc. Mass. Spectrom. 17, 1181-1187 (2006).

6. H.H. Mantsch, D. Chapman, Infrared spectrosc. biomol., (Wiley-Liss, New York, 1996).

7. E. Levenson, P. Lerch, M.C. Martin, Infrared Phys. Tech. 49, 45-52 (2006).

8. Following the method of J. Sambrook, E.F. Fritsch, T. Maniatis, Molecular cloning: $a$ laboratory manual (Cold Spring Harbor Laboratory Press, Cold Spring Harbor, 1989).

9. M. Gottlieb, M. Chavko, Anal. Biochem. 165, 33-37 (1987).

10. J.R. Lawrence et al., Appl. Environ. Microbiol. 69, 5543-5554 (2003).

11. H. Zhang, B. Gilbert, F. Huang, J.F. Banfield, Nature 424, 1025-1029 (2003).

12. A.E. Martell, R.M. Smith, Crit. stability constants, vol. 1: amino acids (Plenum Press, New York, 1974).

13. S. Romer et al., J. Appl. Crystallog. 36, 1-6 (2003).

14. S.W. Provencher, Comput. Phys. Comm. 27, 229-242 (1982). 
1
2
3 


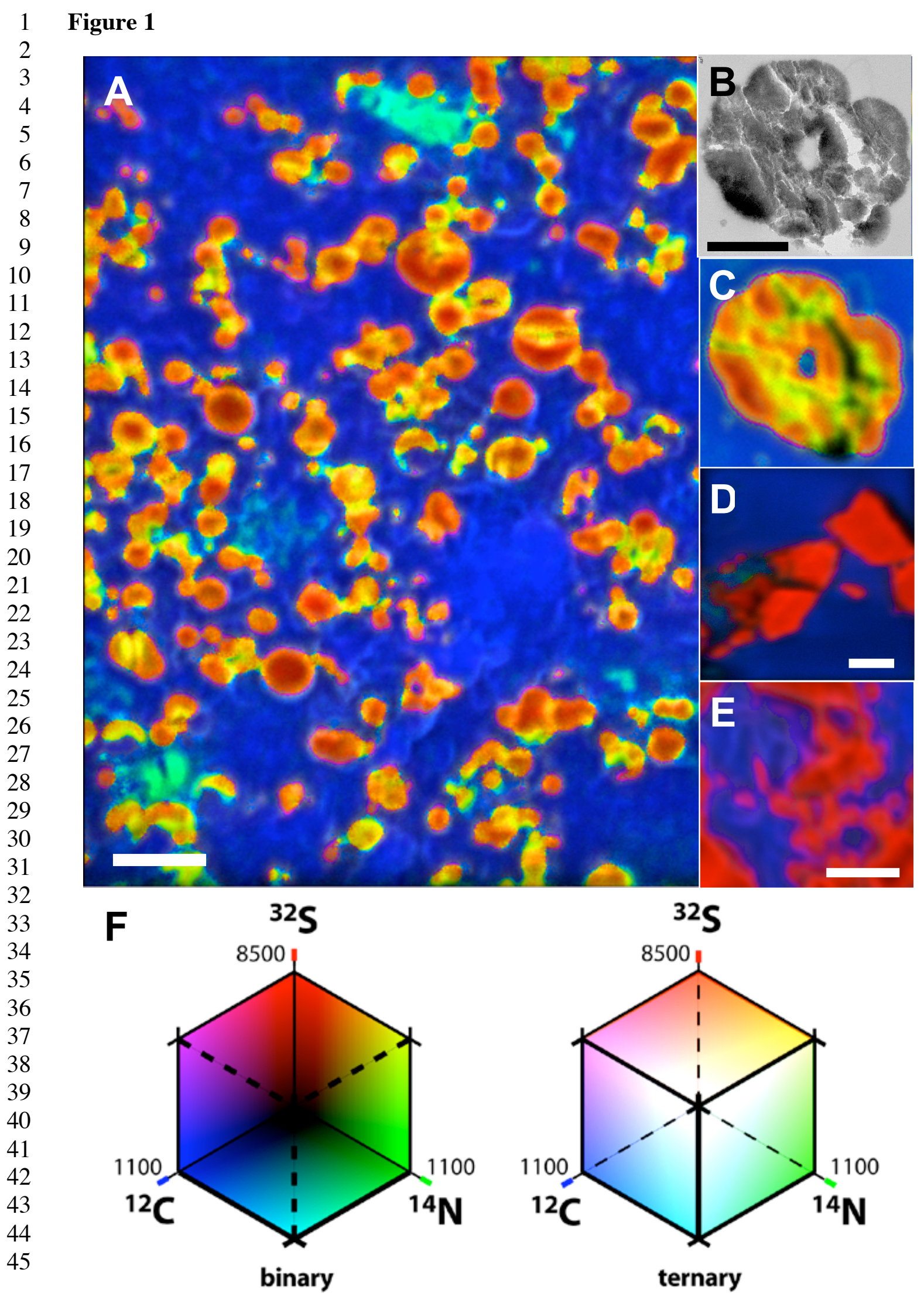


Figure 2

2

3

4

5

6

7
8

9

10

11

12

13

14

15

16

17

18

19

20

21

22

23

24

25

26

27

28

29

30

31

32

33

34

35

36

37

38

39

40

41

42

43

44

45

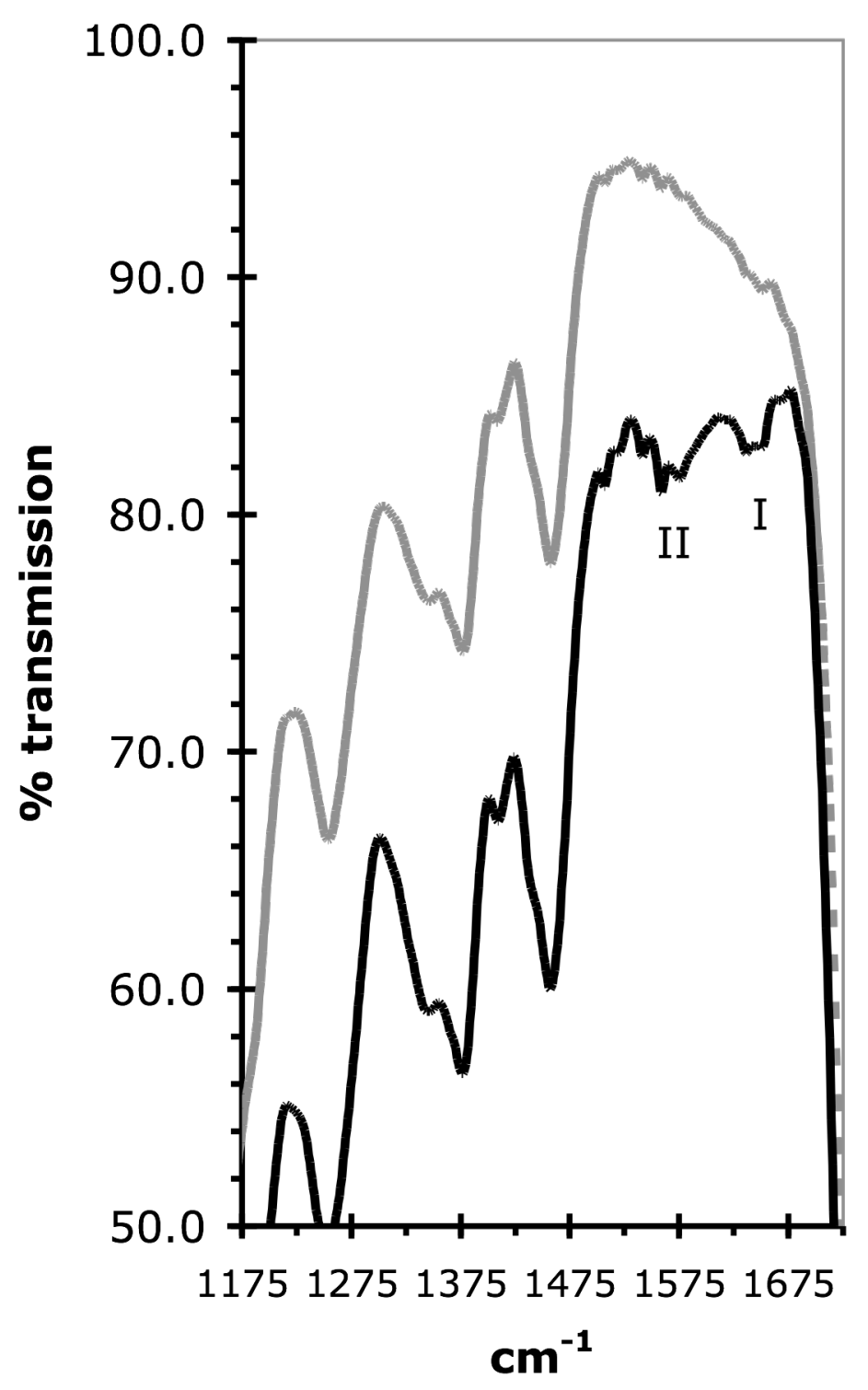


Figure 3

2

3

4

5

6

7

8

9

10

11

12

13

14

15

16

17

18

19

20

21

22

23

24

25

26

27

28

29

30

31

32

33

34

35

36

37

38

39

40

41

42

43

44

45 


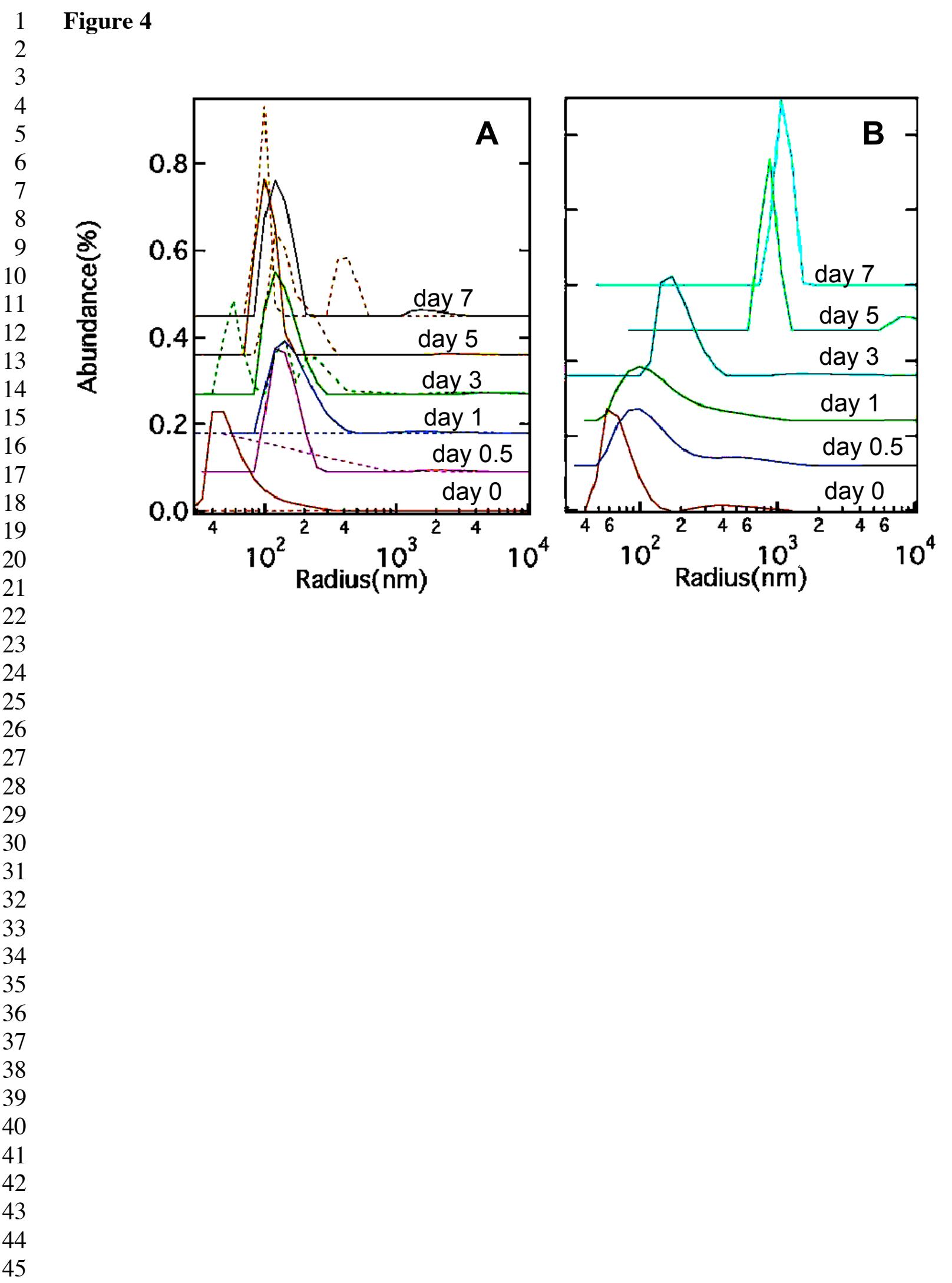

